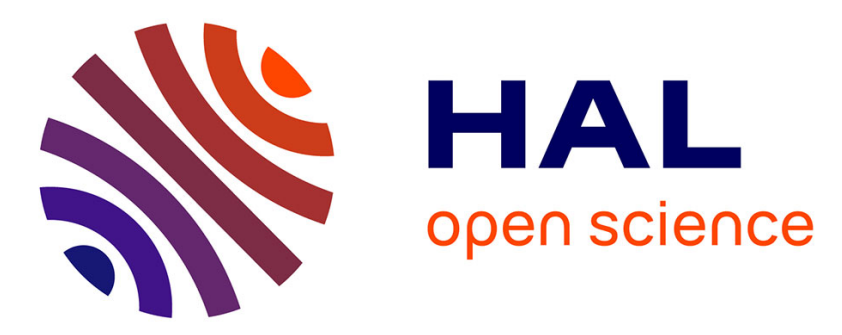

\title{
The ability of individuals to assess population density influences the evolution of emigration propensity and dispersal distance
}

\author{
Hans Joachim Poethke, Andreas Gros, Thomas Hovestadt
}

\section{- To cite this version:}

Hans Joachim Poethke, Andreas Gros, Thomas Hovestadt. The ability of individuals to assess population density influences the evolution of emigration propensity and dispersal distance. Journal of Theoretical Biology, 2011, 10.1016/j.jtbi.2011.05.012 . hal-00709542

\section{HAL Id: hal-00709542 \\ https://hal.science/hal-00709542}

Submitted on 19 Jun 2012

HAL is a multi-disciplinary open access archive for the deposit and dissemination of scientific research documents, whether they are published or not. The documents may come from teaching and research institutions in France or abroad, or from public or private research centers.
L'archive ouverte pluridisciplinaire HAL, est destinée au dépôt et à la diffusion de documents scientifiques de niveau recherche, publiés ou non, émanant des établissements d'enseignement et de recherche français ou étrangers, des laboratoires publics ou privés. 


\section{Author's Accepted Manuscript}

The ability of individuals to assess population density influences the evolution of emigration propensity and dispersal distance

Hans Joachim Poethke, Andreas Gros, Thomas Hovestadt

PII: S0022-5193(11)00254-2

DOI: doi:10.1016/j.jtbi.2011.05.012

Reference: YJTBI 6476

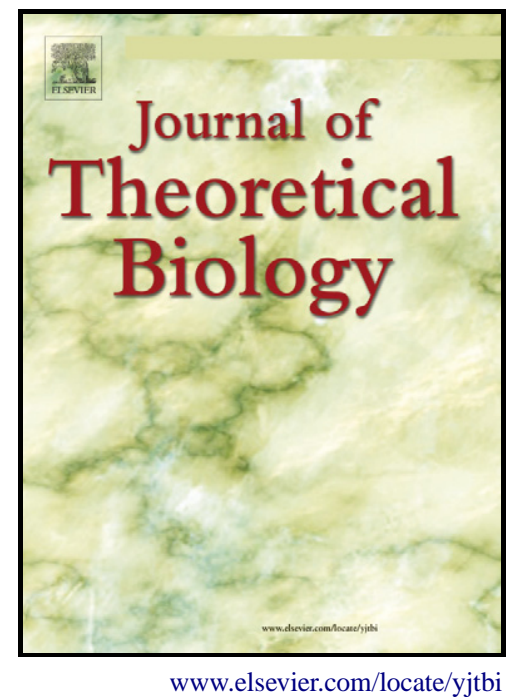

To appear in: Journal of Theoretical Biology

Received date: $\quad 20$ December 2010

Revised date: $\quad 26$ April 2011

Accepted date: 10 May 2011

Cite this article as: Hans Joachim Poethke, Andreas Gros and Thomas Hovestadt, The ability of individuals to assess population density influences the evolution of emigration propensity and dispersal distance, Journal of Theoretical Biology, doi:10.1016/j.jtbi.2011.05.012

This is a PDF file of an unedited manuscript that has been accepted for publication. As a service to our customers we are providing this early version of the manuscript. The manuscript will undergo copyediting, typesetting, and review of the resulting galley proof before it is published in its final citable form. Please note that during the production process errors may be discovered which could affect the content, and all legal disclaimers that apply to the journal pertain. 
1 The ability of individuals to assess population density influences the evolution

2 of emigration propensity and dispersal distance.

3

4 Hans Joachim Poethke ${ }^{1^{*}}$, Andreas Gros ${ }^{1,2, \$}$, and Thomas Hovestadt ${ }^{1,3, \S}$

5

$6 \quad{ }^{1}$ University of Würzburg, Field Station Fabrikschleichach,

7 Glashüttenstrasse 5, 96181 Rauhenebrach, Germany

$8 \quad{ }^{2}$ New England Complex Systems Institute, 238 Main Street, Suite 319, Cambridge, 9 MA 02142. USA

$10{ }^{3}$ Muséum National d'Histoire Naturelle, CNRS UMR 7179, 1 Avenue du Petit Château, 1191800 Brunoy, France

12

13 ** poethke@biozentrum.uni-wuerzburg.de

$14 \$$ andreas@necsi.edu

15 §hovestadt@biozentrum.uni-wuerzburg.de

16

17

18

correspondence should be sent to:

19 Hans J. Poethke

20 Field Station Fabrikschleichach, University of Würzburg, Glashüttenstrasse 5,

21 D-96181 Rauhenebrach, Germany

22 e-mail: poethke@biozentrum.uni-wuerzburg.de 


\section{Abstract}

25 We analyze the simultaneous evolution of emigration and settlement decisions for actively 26 dispersing species differing in their ability to assess population density. Using an individual27 based model we simulate dispersal as a multi-step (patch to patch) movement in a world 28 consisting of habitat patches surrounded by a hostile matrix. Each such step is associated with 29 the same mortality risk. Our simulations show that individuals following an informed strategy, 30 where emigration (and settlement) probability depends on local population density, evolve a 31 lower (natal) emigration propensity but disperse over significantly larger distances - i.e. 32 postpone settlement longer - than individuals performing density-independent emigration. 33 This holds especially when variation in environmental conditions is spatially correlated. Both 34 effects can be traced to the informed individuals' ability to better exploit existing 35 heterogeneity in reproductive chances. Yet, already moderate distance-dependent dispersal 36 costs prevent the evolution of multi-step (long- distance) dispersal, irrespective of the 37 dispersal strategy.

39 Keywords: density dependent, kin-competition, distance-dependent dispersal cost, 40 metapopulation, individual-based model 
41

42

43

44

45

46

47

\section{Introduction}

The evolution of dispersal is driven by the balance between dispersal related cost, e.g. the mortality-risk during transitions or the energy and time spent on dispersal (Rankin and Burchsted 1992, Zera and Denno 1997), and numerous potential benefits. These benefits include avoidance of inter- or intraspecific competition for resources (Lambin et al. 2001, Poethke and Hovestadt 2002), minimization of kin-competition (e.g. Hamilton and May 1977, Comins 1982, Frank 1986, Comins and Hassell 1987, Rousset and Gandon 2002, Kisdi 2004, Poethke et al. 2007) avoidance of inbreeding depression (Bengtsson 1978, Motro 1991, Perrin and Goudet 2001) or coping with the temporal variability of resource availability (Levin et al. 1984, Travis and Dytham 1999, Gandon and Michalakis 2001). The possibility to colonize new habitats, another benefit of dispersal, is critical for the persistence of a species (Alsos et al. 2007).

So far theoretical studies on dispersal of actively moving organisms have mainly focused on the emigration of individuals, while the dispersal process itself and the question how far to disperse has been mostly ignored. Instead, the dispersal distance has been tackled with rather arbitrary assumptions like nearest neighbour (e.g. Travis et al. 1999, Gros et al. 2006) or global (e.g. Poethke and Hovestadt 2002) dispersal. The growing awareness of colonization events through long-distance dispersal in plants (Nichols and Hewitt 1994, Nathan et al. 2002, Bohrer et al. 2005, Nathan 2006, Alsos et al. 2007) has inspired the introduction and investigation of "fat-tailed" dispersal kernels (Higgins and Richardson 1999, Bullock and Clarke 2000, Hovestadt et al. 2001, Austerlitz et al. 2004, Katul et al. 2005) in theoretical studies on seed dispersal. However, dispersal kernels describe the probability distribution of dispersal distances away from a source. The use of dispersal kernels has thus mostly been confined to studies of seed or pollen dispersal, or that of other organisms with passive dispersal (Ezoe 1998, Gros et al. 2006) in continuous landscapes. 
66

67

68

69

Although kernels have occasionally been estimated for actively moving animals (Baguette 2003, Gilbert et al. 2004, Chapman et al. 2007) and certain kernels are implicitly assumed in metapopulation models (Hanski 1994), they appear inappropriate to capture the behaviour of actively moving individuals. Such animals presumably do not leave habitat patches accidentally (Van Dyck and Baguette 2005), but because of a decision to do so. Moreover, they are believed to more or less continuously monitor the status of their surroundings during movement (Nathan et al. 2008). It is likely that they can distinguish between suitable and unsuitable habitat and can assess the expected benefit of further movement (Baker and Rao 2004). Habitat selection of actively moving animals has been object to several theoretical and empirical studies (for a review see Morris 2003). Numerous examples show that animals react not only to habitat quality but also on local population density (Morris 2010). For this reason it is rational to consider dispersal of actively moving organisms as a three phase process with different factors influencing decisions concerning i) emigration, ii) movement and iii) settlement (Clobert et al. 2009).

When suitable habitat is patchily distributed and patches of habitat are embedded into an environment unsuitable for reproduction, animals should continue their movement until they find a suitable patch (Baker and Rao 2004, Stamps et al. 2005). In this case the realized dispersal distance of individuals will crucially depend on the mean distance between patches of suitable habitat (e.g. Hein et al. 2004). Yet, once dispersing individuals have discovered a patch of suitable habitat, they may either settle or decide to continue their search for a new breeding site. In this case, the final dispersal distance of actively moving animals will strongly depend on their perseverance i.e. the probability to continue dispersal after having reached a patch of suitable habitat. It is conceivable that an individual able to estimate the quality of its natal site is also able to assess the quality of a site it has arrived in, and may consequently continue to disperse if the quality of that site is perceived as too low. 
91 We thus propose that dispersal distances should strongly depend on the capability of 92 individuals to use information on habitat quality in their dispersal decision whereas 93 uninformed dispersers should typically not continue dispersal once they have reached a new 94 potential breeding habitat. In the following we expand a previously published simulation 95 model (Poethke and Hovestadt 2002) to test this hypothesis. We compare the evolution of 96 dispersal distances for different degrees of spatial correlation in environmental conditions. 97 Local population density is a critical determinant of intraspecific competition and dispersal is 98 strongly motivated by the search for less populated habitats (Ruxton and Rohani, 1999, Travis 99 et al. 1999, Poethke and Hovestadt 2002). To account for organisms differing in their capacity 100 to assess local population density, we thus implement informed (in this case density 101 dependent) as well as uninformed (density independent) dispersal into the model and compare 102 the effect of either strategy on the evolution of dispersal.

103 More specifically, we test a number of hypotheses. (1) The number of successive dispersal 104 steps decreases with increasing dispersal costs (cf. Murrell et al. 2002). (2) The number of 105 dispersal steps increases with increasing spatial correlation in environmental conditions. With 106 increasing spatial correlation, animals have to disperse further to escape poor conditions (cf. 107 Frank and Wissel 1998, Murrell et al. 2002, Kallimanis et al. 2006). (3) Most importantly, we 108 test the hypothesis that short distance (that from the natal site to the first suitable site 109 encountered) dispersal prevails for uninformed dispersers, while dispersal events that cover 110 more than one step will only be found - in significant proportion - for the case of informed 111 (density dependent) dispersal.

\section{2. The model}

114 We use a modified version of the individual-based metapopulation model of Poethke and 115 Hovestadt (2002). The model simulates the population dynamics, inter-patch dispersal, and 
116 the evolution of dispersal propensity in a meta-population of a diploid, sexually reproducing

117 species with discrete generations and density-dependent local population growth. The

118 metapopulation consists of habitat patches arranged in a square lattice of $24 \times 24=576$ cells. To

119 avoid edge effects the metapopulation is closed into a torus (edges are wrapped). Each patch

120 (i) supports a local population with carrying capacity $K=40$; total carrying capacity of the

121 system is thus ca. 23.000 individuals. Simulation experiments are initialised by placing $K$

122 individuals in each of the 576 patches.

123 Within each patch populations grow according to a logistic growth model suggested by

124 Hassell et al. (1976). Similar to Murrell et al. (2002) and Poethke and Hovestadt (2002), a

125 female gives birth to $2 \times \lambda$ offspring where $\lambda$ is Poisson distributed with mean $\lambda_{\text {mean }}(t, i)$. To

126 account for environmental variability, $\lambda_{\text {mean }}(t, i)$ (specific for patch $i$ at timestep $t$ ) is drawn

127 from a log-normal distribution with mean $\bar{\lambda}=2$ and a standard deviation of $\sigma=1$.

128 Offspring are randomly allocated to either sex. Offspring survival during maturation is

129 dependent on the total number of offspring hatched in a patch $\left(N_{i}\right)$. Thus, offspring mature

130 with a density dependent survival probability $s$ where $s=K_{i} /\left[K_{i}+(\bar{\lambda}-1) \cdot N_{i}\right]$.

131 In all of our simulations, individuals are characterized by four alleles $\left(n_{1}, n_{2}, f_{1}, f_{2}\right)$ at two

132 diploid loci $(n, f)$. The first of these loci $(n)$ determines the individual's propensity for natal

133 dispersal $\left(P_{n}\right)$, the second $(f)$ determines its perseverance, that is the probability $\left(P_{f}\right)$ of

134 continuing to disperse once it has successfully reached a suitable habitat patch. These alleles

135 take continuous values and are initialised with uniformly distributed random numbers within

136 the interval $[0.3,0.5]$. Preliminary simulations showed that similar dispersal traits evolved

137 independently of the starting values taken for initialising alleles (see appendix figure A2).

138 Dispersal "decisions" can be either density-dependent (DD) or density-independent (DI). We

139 performed simulation experiments for three different scenarios differing in the assumptions

140 concerning the animals' capacity to perceive local population density and to adjust their 
141 dispersal behaviour accordingly. In the first scenario (scenario $\mathrm{DI}_{\mathrm{n}} / \mathrm{DI}_{\mathrm{f}}$ ) natal dispersal

142 propensity as well as perseverance, i.e. dispersal propensity for all further dispersal moves, is

143 density-independent. In the second scenario $\left(\mathrm{DD}_{\mathrm{n}} / \mathrm{DI}_{\mathrm{f}}\right)$ we assume that individuals can asses

144 the population density in their natal patch, but do not have ability to assess the density of any

145 patch they reach thereafter. Thus, natal dispersal propensity is density-dependent $\left(\mathrm{DD}_{\mathrm{n}}\right)$ but

146 perseverance is density-independent $\left(\mathrm{DI}_{\mathrm{f}}\right)$. In the third scenario $\left(\mathrm{DD}_{\mathrm{n}} / \mathrm{DD}_{\mathrm{f}}\right)$ all dispersal steps

147 are density-dependent.

148 In the case of density independent dispersal, dispersal probability (the phenotype) is

149 calculated according to the mean value of both alleles at the respective loci. This holds for

150 density independent natal dispersal probability $\left(P_{D I, n}=\left(n_{1}+n_{2}\right) / 2\right)$ as well as for the

151 probability to continue dispersal $\left(P_{D I, f}=\left(f_{1}+f_{2}\right) / 2\right)$ in the two scenarios with density

152 independent perseverance $\left(\mathrm{DI}_{\mathrm{n}}, \mathrm{DI}_{\mathrm{f}}\right.$ and $\left.\mathrm{DD}_{\mathrm{n}}, \mathrm{DI}_{\mathrm{f}}\right)$.

153 In the case of density dependent natal dispersal, individual alleles do not determine the

154 dispersal probability but a threshold density $C_{n}=\left(n_{1}+n_{2}\right) / 2$ for dispersal. The actual dispersal 155 probability $P_{D D, n}$ is then calculated according to the model derived by Poethke and Hovestadt $156 \quad(2002)$ as

$$
P_{D D, n}=\left\{\begin{array}{c}
0 \quad \text { if } \quad C_{i}<C_{n} \\
1-\frac{C_{n}}{C_{i}} \quad \text { if } \quad C_{i} \geq C_{n}
\end{array}\right.
$$

158 Accordingly, the probability to continue dispersal in the case of density dependent 159 perseverance $\left(\mathrm{DD}_{\mathrm{n}}, \mathrm{DD}_{\mathrm{f}}\right)$ is calculated as

$$
P_{D D, f}=\left\{\begin{array}{c}
0 \quad \text { if } \quad C_{i}<C_{f} \\
1-\frac{C_{f}}{C_{i}} \quad \text { if } \quad C_{i} \geq C_{f}
\end{array}\right.
$$
with $C_{f}=\left(f_{1}+f_{2}\right) / 2$

162 For a full discussion concerning the adequacy of this model structure for density dependent 
163 dispersal see Poethke and Hovestadt (2002) and Hovestadt et al (2010). It should be noted

164 that Ruxton and Rohani (1998) already utilized a model very similar to our $\mathrm{DD}_{\mathrm{n}} / \mathrm{DD}_{\mathrm{f}}$ model

165 version to address questions concerning the stabilizing effects of dispersal on population 166 dynamics.

167 The three scenarios may correspond to three types of dispersers with different sensory 168 capacities. The first scenario $\left(\mathrm{DI}_{\mathrm{n}} / \mathrm{DI}_{\mathrm{f}}\right)$ could apply to animals such as certain butterflies that 169 leave habitats independent of population density (e.g. Boughton 2000). The second scenario $170\left(\mathrm{DD}_{\mathrm{n}} / \mathrm{DI}_{\mathrm{f}}\right)$ may apply to animals that acquire important information about population density 171 during their larval stage but disperse regardless of density as adults, such as e.g., migratory 172 locusts (Fuchs et al. 2003), or some crickets (Fowler 1988). In the third setting $\left(\mathrm{DD}_{\mathrm{n}} / \mathrm{DD}_{\mathrm{f}}\right)$ we 173 assume animals that have the ability to assess local population density at any time. This most 174 likely applies to long-lived animals like mammals, birds, and a few insect species, e.g. some 175 corixid species investigated by Pajunen and Pajunen (2003).

176 For natal dispersal an individual randomly chooses one of eight neighbouring patches as 177 destination and then assesses successive patches along this trajectory thus keeping its 178 direction in each following step (see Figure 1). Dispersing individuals either reach the next 179 habitat patch or die during the transition with probability $\mu$. Exploratory simulation runs 180 showed that individuals very rarely continued their dispersal for more than 10 consecutive 181 steps (see also results section), and we therefore limited the maximum number of movement 182 steps to 15 . This had a negligible effect on simulation results but significantly reduced 183 computation time.

184 We modelled spatial correlation in habitat quality by aggregating patches into clusters and 185 assigning an identical value of $\lambda_{\text {mean }}(t, i)$ to all $k$ patches within such a cluster. To account for 186 different spatial dimensions of correlation we vary the side-length of clusters from $l=1$ 187 (cluster-size $k=1$; no spatial correlation) to $l=4$ (cluster-size $k=16$; see fig 1 ). Reproductive 
188 conditions $\left(\lambda_{\text {mean }}(t, i)\right)$ for each cluster were drawn anew every generation $(t)$. The spatial 189 configuration of clusters does not change over time, so that each patch always belongs to the 190 same cluster.

191 When inheriting its parent's genes, mutations occur with a small probability $(m=0.01)$ per

192 allele. If mutations occur, the affected alleles are altered by adding a random value drawn 193 from a uniform distribution within the interval $[-0.1,0.1]$. Preliminary simulation

194 experiments showed that an evolutionary equilibrium is usually reached after approximately

1953000 generations (see appendix figure A2), yet each simulation run included 10000 196 generations. All results were recorded in the last generation. We varied stepwise dispersal 197 mortality $\mu$ as follows: $\mu=\{0.025,0.05,0.10$, and 0.20$\}$.

\section{Results}

In all scenarios we find that uninformed dispersers $\left(\mathrm{DI}_{\mathrm{n}}\right)$ evolve higher probabilities of natal

201 dispersal than informed dispersers $\left(\mathrm{DD}_{\mathrm{n}}\right.$; fig. 2a), whilst consecutive dispersal steps occur

202 with higher probability for informed dispersers $\left(\mathrm{DD}_{\mathrm{f}}\right)$ than for uninformed dispersers $\left(\mathrm{DI}_{\mathrm{f}}\right.$; fig.

203 2b). Not surprisingly, natal dispersal probability (fig. 2a) as well as the mean number of

204 additional steps moved (fig. 2b) decreases rapidly with increasing dispersal mortality $(\mu)$. In

205 general, individuals are thus inclined to settle in the first patch they arrive in as soon as

206 dispersal mortality rises beyond even moderate levels $(\mu \geq 0.1)$. The effect of dispersal

207 mortality on natal dispersal is more pronounced for the case of density independent natal

208 dispersal than for informed dispersal (fig. 2a).

209 Evolution of elevated natal dispersal probabilities for uninformed ( $\mathrm{DI}_{\mathrm{n}}$, density independent)

210 compared to informed natal dispersal $\left(\mathrm{DD}_{\mathrm{n}}\right)$ has already been described by Enfjall and Leimar

211 (2009) and Hovestadt et al. (2010): density dependent dispersal more efficiently exploits

212 inter-patch differences in population density, but also more efficiently homogenizes 
213 population density across patches. To demonstrate this figure 3 compares the demographic

214 benefits, that is, the mean difference in population size between the source and destination

215 patch for the first five dispersal steps of emigrants. Evidently, the very first dispersal step is

216 the most profitable one, i.e. is associated with the largest decline in pre- (natal patch)

217 compared to post-dispersal (target patch) density. This holds for density dependent as well as

218 for density independent dispersal. For example, with low dispersal cost $(\mu=0.025)$ and an

219 accordingly high emigration probability, uninformed natal dispersers $\left(\mathrm{DI}_{\mathrm{n}} / \mathrm{DI}_{\mathrm{f}}\right)$ arrive after

220 their first dispersal step in a patch with on average 10.8 fewer competitors than in their natal

221 patch (full dots, fig. 3a). If these individuals continue to disperse their second step will bring

222 them to a patch with on average only 1.1 fewer individuals then their first target. With higher

223 dispersal cost $(\mu=0.200)$ emigration probabilities become generally lower but,

224 correspondingly, the demographic benefits of natal dispersal increases (fig. 3b). Thus, with

225 higher dispersal mortality $(\mu=0.200)$ uninformed natal dispersers $\left(\mathrm{DI}_{\mathrm{n}} / \mathrm{DI}_{\mathrm{f}}\right)$ arrive after their

226 first dispersal step in a patch with on average 13.7 fewer individuals than in their natal patch

227 (full dots, fig. 3b).

228 Informed dispersal allows a more efficient exploitation of inter-patch differences in

229 population density. Even for the case of low dispersal cost (and relatively high emigration

230 probability), density dependent natal dispersers $\left(\mathrm{DD}_{\mathrm{n}} / \mathrm{DI}_{\mathrm{f}}\right.$ and $\left.\mathrm{DD}_{\mathrm{n}} / \mathrm{DD}_{\mathrm{f}}\right)$ arrive in patches with

231 on average 22.8 fewer individuals than in their natal patch (grey dots and open dots in fig. 3a).

232 For the case of high dispersal cost $(\mu=0.200)$ the mean reduction in local population size

233 achieved increases to 38.6 individuals (grey dots and open dots in fig. 3b).

234 The difference in the potential demographic benefit gained by dispersal between informed and

235 uninformed strategies is not restricted to the first dispersal step: it is even more pronounced

236 for the steps following natal dispersal. While the mean demographic benefit of a second

237 dispersal step (i.e. the mean difference in population density between the target of their first 
238 (natal) dispersal step and the target of their second step) for completely uninformed dispersers

$239\left(\mathrm{DI}_{\mathrm{n}} / \mathrm{DI}_{\mathrm{f}}\right)$ is only 1.1 individuals (largely independent of dispersal cost, see filled circles in fig.

$2403 \mathrm{a}$ and fig. 3b), completely informed dispersers $\left(\mathrm{DD}_{\mathrm{n}} / \mathrm{DD}_{\mathrm{f}}\right)$ will realize a mean demographic

241 benefit of 5.2 individuals in the case of low dispersal cost $(\mu=0.025$; open circles in fig. 3a)

242 and of 12.1 individuals for high dispersal cost $(\mu=0.200$; open circles in fig. $3 b)$ with a

243 second dispersal step. Even those individuals that show informed dispersal in their first step

244 but density independent dispersal in all other steps $\left(\mathrm{DD}_{\mathrm{n}} / \mathrm{DI}_{\mathrm{f}}\right.$, grey circles) do profit slightly

245 more from steps following natal dispersal than completely uninformed dispersers.

246 Natal dispersal equalizes population density to a considerable degree, thus reducing the 247 potential benefits of further steps, while the costs for any such step remain the same.

248 Consequently, even in scenarios with a rather benign cost of dispersal (e.g. $\mu=0.1$ ), only $2-5 \%$

249 of the emigrants take more than one dispersal step. With spatially correlated environmental 250 fluctuations (figs. 3c and 3d) however, the demographic benefit for natal dispersal decreases 251 while the benefit for all further dispersal steps increases. An increase in the demographic 252 benefits gained by moving additional steps particularly emerges for individuals following 253 uninformed secondary dispersal (for $\mathrm{DI}_{\mathrm{n}} / \mathrm{DI}_{\mathrm{f}}$ compare filled dots in figs. $3 \mathrm{a}$ and b with figs $3 \mathrm{c}$ 254 and $\mathrm{d}$; for $\mathrm{DD}_{\mathrm{n}} / \mathrm{DI}_{\mathrm{f}}$ compare grey dots in figs. 3a and b with figs. $3 \mathrm{c}$ and d) and it is largest for 255 individuals that show density dependent natal dispersal but density independent dispersal in 256 all other dispersal steps $\left(\mathrm{DD}_{\mathrm{n}} / \mathrm{DI}_{\mathrm{f}}\right)$.

257 Spatial correlation has only a negligible effect on the probability of natal dispersal (fig. 4a) 258 but the number of additional dispersal steps taken is strongly influenced by the spatial 259 correlation in environmental conditions (fig. 4b); here we only show results for scenario $260 \mathrm{DI}_{\mathrm{n}} / \mathrm{DI}_{\mathrm{f}}$ but results for the two other strategies are analogous (see appendix). At low dispersal 261 mortality $(\mu=0.025)$ the mean number of dispersal steps increases from 1.08 to 1.58 as cluster 262 dimension increases from $k=1$ to $\mathrm{k}=16$. Nonetheless, only $34 \%$ of all emigrants will continue 
263 dispersal after the first dispersal step (see black dots in fig. A1). However, at a dispersal

264 mortality of $\mu=0.1$, the mean number of dispersal steps increases only from 1.02 to 1.05 .

\section{Discussion}

The results of our evolutionary simulation experiments show that organisms with active

269 dispersal should typically settle into the first patch of suitable habitat they encounter, i.e.

270 disperse over short distances. Even for rather low levels of dispersal mortality individuals

271 hardly ever continue dispersal once they have reached a suitable patch. This supports the

272 findings by Barton et al. (2009), who modelled the (evolution of) movement rules of

273 organisms during transition. They found that organisms should target their movement to the

274 first (nearest) patch when patches become rare and movement in the matrix thus becomes

275 more costly. Selection for settlement in the first patch encountered particularly evolves for

276 uninformed dispersers and for spatially uncorrelated environmental conditions. Yet even in

277 spatially correlated environments density independent dispersers mostly settle in the

278 neighbouring patch as soon as step-wise dispersal mortality becomes moderate (e.g. $\mu \geq 0.1$ ).

279 Thus, most of these dispersers did not even leave the range of environmental correlation in the

280 scenarios with extended spatial clusters.

281 Under density dependent natal dispersal, the general tendency of individuals to leave the natal

282 patch decreases. However, at the same time, the average dispersal distance (number of

283 consecutive dispersal steps) is increased. Thus, the largest mean number of dispersal steps

284 recorded emerges for completely informed dispersal $\left(\mathrm{DD}_{\mathrm{n}} / \mathrm{DD}_{\mathrm{f}}\right)$ in spatially correlated

285 landscapes. However, even in these scenarios, individuals typically move less than two steps

286 as soon as stepwise mortality risk becomes moderately large $(\mu \geq 0.1)$.

287 As the costs for any dispersal step remain the same in our simulations, this decline in dispersal 
288 probability must be attributed to a declining benefit of additional dispersal steps as the number

289 of steps increases. This declining benefit has two main causes: (1) Kin competition, a major

290 driver of dispersal (Hamilton and May 1977, Rousset and Gandon 2002, Poethke et al. 2007),

291 is already largely reduced with the first dispersal step, because siblings are spread over eight

292 neighbouring patches. Note that individual based simulation models of the evolutionary

293 process account for kin competition by default (Poethke et al. 2007). (2) The chance to reach a

294 habitat with fewer competitors decreases with every dispersal step simply because any round

295 of dispersal homogenizes population density ever further. The fact that spatial correlation has

296 little effect on natal emigration probabilities (see fig. 4a) despite the fact that demographic

297 benefits decline, supports the notion that this step is to a large degree driven by avoidance of

298 kin-competition. Only selection on taking further steps seems to be sensitive to demographic

299 benefits. The ability to control these latter benefits - moving from high- to low-density

300 populations - is responsible for the significant differences between informed and uninformed

301 dispersal strategies.

302 The evolution of elevated dispersal probabilities for uninformed ( $\mathrm{DI}_{\mathrm{n}}$, density independent)

303 compared to informed natal dispersal $\left(\mathrm{DD}_{\mathrm{n}}\right)$ can be traced to the fact that density dependent

304 dispersal more efficiently exploits inter-patch differences in population density, but also more

305 efficiently homogenizes population density across patches (Enfjall and Leimar 2009,

306 Hovestadt et al. 2010). This is the flip side of the larger demographic benefit achieved by

307 dispersing individuals following a density-dependent emigration strategy (fig. 3). Clearly,

308 homogenizing occurs most rapidly when the overall number of emigrants is large, i.e. when

309 dispersal mortality is low.

310 Only the DD strategy is adjustable in a way that allows 'compensating' increased dispersal

311 costs by also increasing the benefits as individuals emigrate only from especially densely

312 populated populations and thus collect especially large demographic fitness benefits given 
313 they survive dispersal (Poethke et al. 2007). This explains the fact that under density-

314 dependent emigration natal dispersal is rarer, but consecutive dispersal steps occur with

315 higher probability.

316 Even when individuals disperse with a fixed probability $\left(\mathrm{DI}_{\mathrm{n}}\right)$, more natal emigrants come, for

317 simple statistical reasons, from high density patches. Apart from the effect of kin-competition

318 (Hamilton and May 1977, Poethke et al. 2007), it is this simple truism that makes even

319 density independent natal dispersal a beneficial strategy in landscapes that exhibit spatio-

320 temporal variability in population density. For dispersal steps following natal dispersal this

321 argument does not hold however. Only immigrants, i.e. individuals that previously dispersed

322 already, are allowed to decide whether they want to continue dispersal or not, but a correlation

323 between population density and the number of immigrants no longer exists.

324 On the other hand, with completely density dependent dispersal $\left(\mathrm{DD}_{\mathrm{n}} / \mathrm{DD}_{\mathrm{f}}\right)$ immigrants can

325 'recognize' that they have arrived in a high-density patch and decide to move on. The

326 probability that this is indeed the case is supported by another mechanism already described

327 by (Hovestadt and Poethke 2006): under density dependent dispersal the distribution of the

328 number of emigrants becomes much more leptokurtic with occasional 'mass emigration' from

329 patches that have particularly good years. Such events also imply that large numbers of

330 individuals immigrate into the neighbouring patches. Consequently, dispersal itself creates a

331 spatial correlation in population density and makes it more profitable to continue dispersal

332 over several steps to leave the zone of correlation in population density. It is indeed this later

333 effect that explains why, rather surprisingly, the mixed strategy $\left(\mathrm{DD}_{\mathrm{n}} / \mathrm{DI}_{\mathrm{f}}\right)$ also evolves higher

334 probabilities for consecutive dispersal steps. Due to the aforementioned effect, the recognition

335 of high density in the natal patch allows already predicting that, after the first 'round' of

336 dispersal, population density in neighbouring patches will be large too, even if individuals do

337 not have the ability to perceive the actual density there. 
338 In our model we assume that individuals either have information on local population density

339 or not and that information acquisition is not costly. In real systems information acquisition

340 may come with a cost, e.g. as investment into sensual capabilities or into time needed for

341 information acquisition. Thus animals often use environmental cues (e.g. Stamps and

342 Krishnan 2005, Ichiki et al 2011) or information derived from the presence of heterosopecifics

343 (e.g. Monikkonen et al. 1999) to assess local habitat quality. Such indirect information will

344 necessarily be less precise. Thus, situations of intermediate information status will exist, too.

345 It requires further investigation to understand how information precision and investment into

346 its acquisition would affect the evolution of conditional dispersal strategies.

347 Despite the enormous interest of theoretical ecologists in the evolution of dispersal and its

348 ecological consequences, the great majority of studies on dispersal evolution have so far

349 focused on the evolution of dispersal propensity (e.g. Hamilton and May 1977, Janosi and

350 Scheuring 1997, Travis and Dytham 1999, Travis et al. 1999, Poethke and Hovestadt 2002,

351 Parvinen et al. 2003), while a smaller number of papers investigate the evolution of dispersal

352 distance (e.g. Ezoe 1998, Savill and Hogeweg 1998, 1999, Hovestadt et al. 2001, Murrell et

353 al. 2002, Rousset and Gandon 2002, Higgins et al. 312 2003, Muller-Landau et al. 2003,

354 Starrfelt and Kokko 2010, Travis et al 2010).

355 Usually the evolution of dispersal distances and the prediction of evolutionarily stable

356 dispersal kernels are analysed under the assumption of 'passive' and uncontrollable movement

357 as it occurs in seed dispersal, while investigating dispersal propensity has been a focus of

358 animal ecologists. At least in part this dichotomy is explained by the different mechanisms

359 that govern dispersal in plants and animals. In active as well as in passive dispersers, dispersal

360 incurs different types of cost. First, species that belong to either dispersal group must invest in

361 equipment that allows dispersal. This may be the flight apparatus of wind dispersed seeds

362 (Tackenberg et al. 2003), the elaiosoma of ant dispersed seeds (Pfeiffer et al. 2010), the wings 
363 and flight muscles of Orthoptera (e.g. Zera and Harshman 2001), or the silk thread of spiders

364 (e.g. Bonte et al. 2003). Such investment costs are paid once and are more or less independent

365 of the distance an individual will ultimately travel. However there are further costs that are

366 related to dispersal distance. With passive dispersal (especially in plants) the number of

367 propagules produced is typically large and dispersal is under maternal control. From the

368 perspective of a tree, it is the overall distribution of offspring that counts and it may pay in

369 evolutionary terms to allocate at least some offspring to risky long-distance dispersal. This

370 promotes the evolution of "fat tailed" dispersal kernels (Hovestadt et al. 2001; see also Roy et

371 al. 2001, Rousset and Gandon 2002, Starrfelt and Kokko 2010).

372 However, as explained in the introduction these approaches appear ill suited for the case of 373 actively moving organisms where the dispersal distances observed should be traced to the 374 interaction between landscape attributes and the rules governing the movement and especially 375 the settlement of organisms. Actively moving animals may continuously monitor their 376 surroundings (Getz and Saltz 2008, Nathan et al. 2008) and decide whether they continue to 377 move or stay once they have found a patch of habitat. At this moment the dispersing 378 individual may decide to either settle or move on. According to our results the tendency to 379 move on will clearly depend on the risk associated with searching for other suitable habitats 380 (cost of dispersal). In addition we would predict that species with well developed sensory (and 381 cognitive) abilities that are readily able to assess population density or habitat quality are 382 more likely to move on than species that do not have such abilities. On the other hand, 383 individuals that need much time (in relation to life-expectancy) or energy to collect such 384 information would typically settle in the first habitat they find.

385 Dispersal distance is an emergent property depending on behavioural rules on the one hand 386 and landscape attributes on the other (cf. Baguett and Van Dyck 2007). As 'smart' animals are 387 unlikely to settle just somewhere in the 'matrix' the distribution of dispersal distances will 
388 strongly be defined by the typical distances between patches of suitable habitat. We believe

389 that our, certainly simplifying approach, is a useful step to better understand the evolution of

390 dispersal distances in actively moving organisms as an emergent property defined by

391 landscape attributes on the one hand and settlement rules on the other.

392 In summary we predict that with uninformed dispersal multiple dispersal steps should evolve

393 only if step-wise dispersal costs are rather low. Multiple dispersal steps are most likely to

394 evolve in species able to assess the quality of at least their natal habitat and in landscapes that

395 show strong spatial correlation of environmental conditions. However, as it presumably

396 requires time to collect information about habitat quality we expect that secondary dispersal

397 will most likely occur among long-lived species with considerable sensory and cognitive

398 abilities like mammals or birds.

\section{Acknowledgements}

401 This work was supported by funding of the German Science Foundation to HJP and TH (DFG

402 PO 244/3-1) and by the DLR (project TenLamas, EU FP6 BiodivERsA Eranet) to TH. We

403 thank Joseph Chipperfield and two anonymous reviewers for their helpful comments on this

404 manuscript.

405 


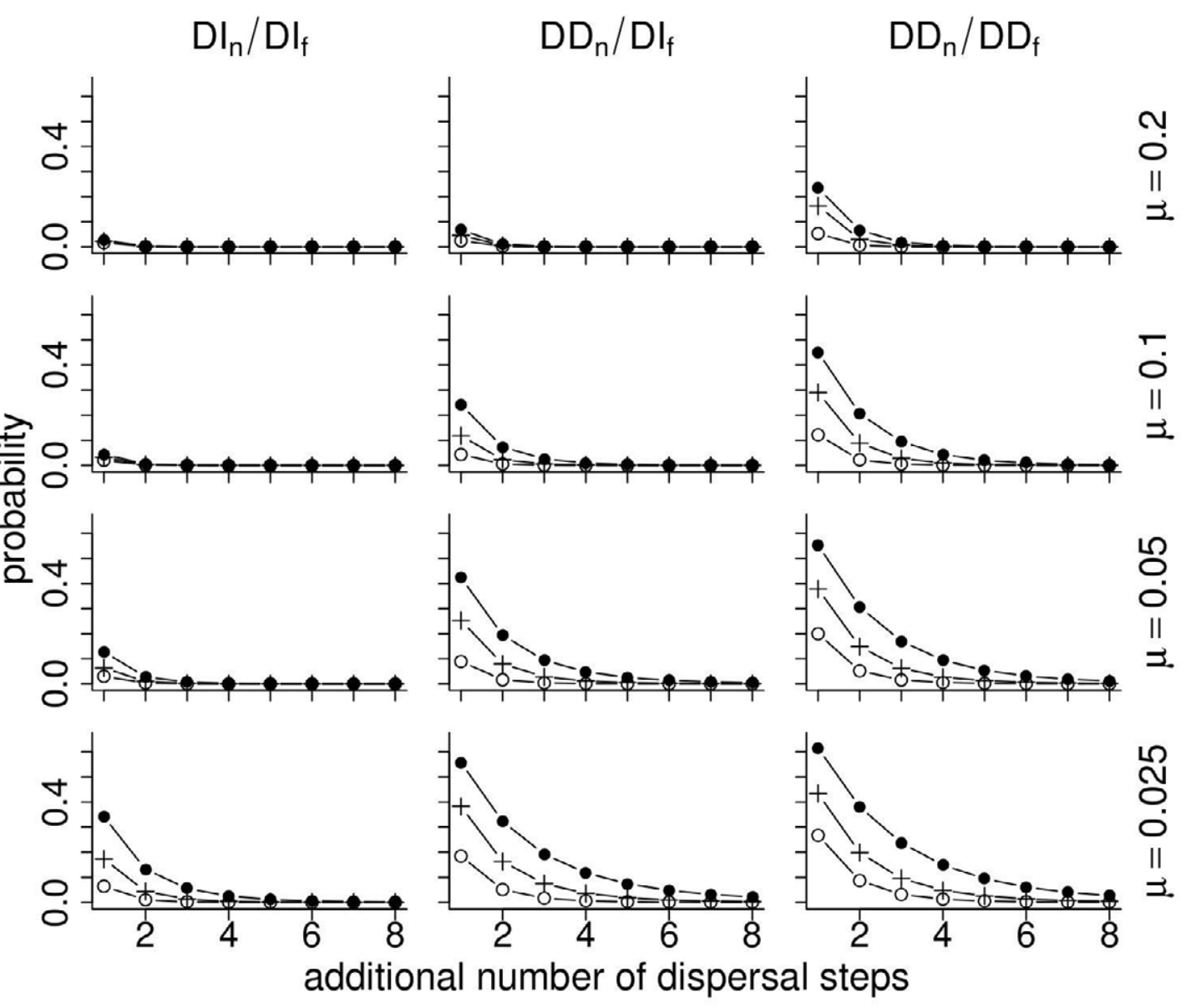

408

Figure A1

410 Probability to perform at least $n$ additional dispersal steps following initial natal dispersal,

411 with $n$ plotted on $x$-axis, as a function of dispersal cost ( $\mu$; rows) and the mode of dispersal

412 (columns). $\mathrm{DI}_{\mathrm{n}} / \mathrm{DI}_{\mathrm{f}}$ : completely uninformed dispersal, $\mathrm{DD}_{\mathrm{n}} / \mathrm{DI}_{\mathrm{f}}$ : density dependent natal

413 dispersal but uninformed dispersal in all subsequent dispersal steps. $\mathrm{DD}_{\mathrm{n}} / \mathrm{DD}_{\mathrm{f}}$ : completely

414 density dependent dispersal. Different symbols reflect results for different scales of spatial

415 correlation. Open circles: results for completely uncorrelated environmental conditions

416 (cluster-size $k=1$ ), crosses for clusters of $k=4$ patches, and filled circles for clusters of $k=16$

417 patches. In any generation, patches within same cluster are exposed to identical environmental 418 conditions $\left(\lambda_{t}\right)$. 
a)

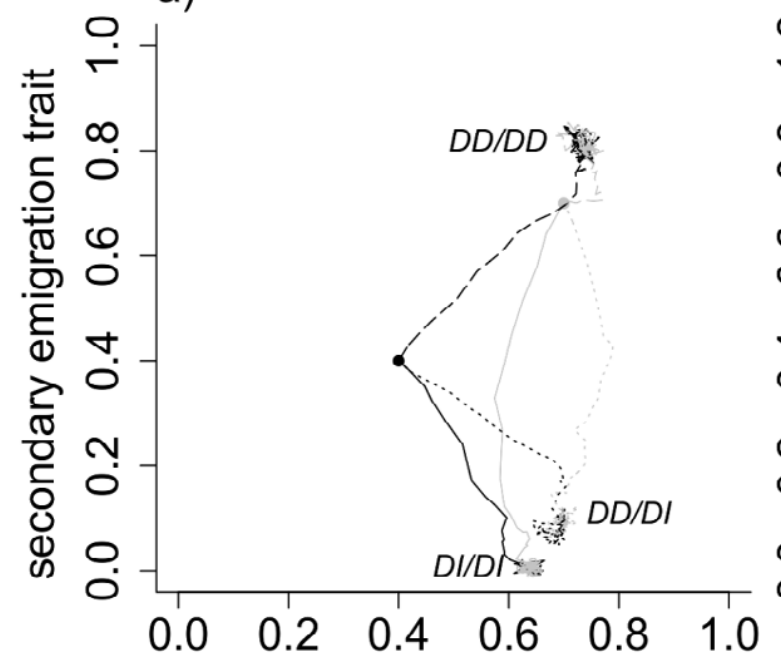

b)

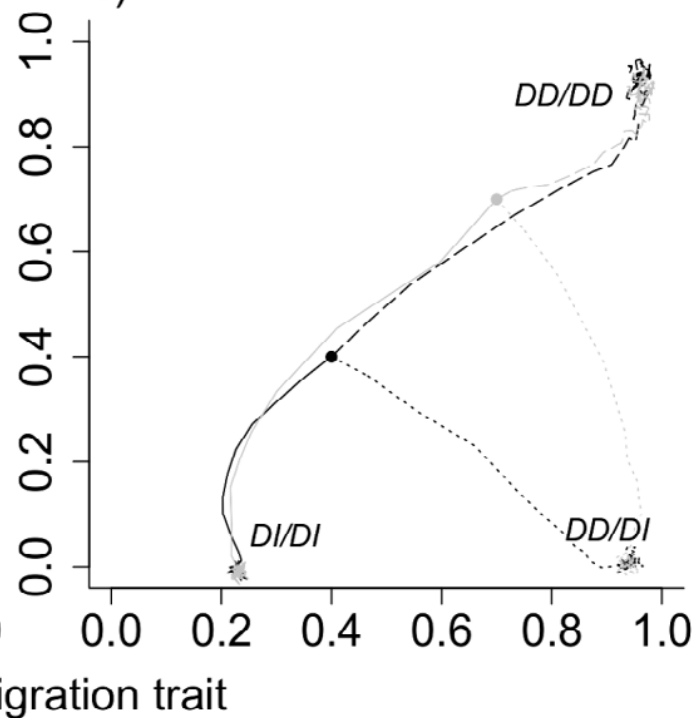

Figure A2

423 Exemplary plots of evolutionary trajectories for different dispersal mortalities (a: $\mu=0.05$ and b: $\mu=0.20$ ) and for each of the three different dispersal strategies investigated: $\mathrm{DI}_{\mathrm{n}} / \mathrm{DI}_{\mathrm{f}}$ 425 (continuous lines), $\mathrm{DD}_{\mathrm{n}} / \mathrm{DI}_{\mathrm{f}}$ (dotted lines), and $\mathrm{DD}_{\mathrm{n}} / \mathrm{DD}_{\mathrm{f}}$ (hatched lines). For each strategy we 426 initialized simulations at two different trait values for the two evolving traits (black and grey 427 dot). Note that the trait evolving is emigration probability for density-independent emigration 428 (DI) but the density threshold ( $C_{n}$ and $C_{f}$ of equation1) for density-dependent emigration 429 (DD). Emigration probabilities for the latter are shown in figures 2 and 4. 
432

433

434

435

436

437

438

439

440

441

442

443

444

445

446

447

448

449

450

451

452

453

454

455

\section{Literature}

Alsos, I. G., P. B. Eidesen, D. Ehrich, I. Skrede, K. Westergaard, G. H. Jacobsen, J. Y. Landvik, P. Taberlet, and C. Brochmann. 2007. Frequent long-distance plant colonization in the changing Arctic. Science 316:1606-1609.

Austerlitz, F., C. W. Dick, C. Dutech, E. K. Klein, S. Oddou-Muratorio, P. E. Smouse, and V. L. Sork. 2004. Using genetic markers to estimate the pollen dispersal curve. Molecular Ecology 13:937-954.

Baguette, M. 2003. Long distance dispersal and landscape occupancy in a metapopulation of the cranberry fritillary butterfly. Ecography 26:153-160.

Baguette, M. and H. Van Dyck. 2007. Landscape connectivity and animal behavior: functional grain as a key determinant for dispersal. Landscape Ecology 22:1117-1129.

Baker, M. B. and S. Rao. 2004. Incremental costs and benefits shape natal dispersal: Theory and example with Hemilepistus reaumuri. Ecology 85:1039-1051.

Barton, K. A., B. L. Phillips, J. M. Morales, and J. M. J. Travis. 2009. The evolution of an 'intelligent' dispersal strategy: biased, correlated random walks in patchy landscapes. Oikos 118:309-319.

Bengtsson, B. O. 1978. Avoiding inbreeding - at what cost? Journal of Theoretical Biology 73:439-444.

Bohrer, G., R. Nathan, and S. Volis. 2005. Effects of long-distance dispersal for metapopulation survival and genetic structure at ecological time and spatial scales. Journal of Ecology 93:1029-1040.

Bonte, D., I. Deblauwe, and J. P. Maelfait. 2003. Environmental and genetic background of tiptoe-initiating behaviour in the dwarfspider Erigone atra. Animal Behaviour 66:169174. 
456

457

458

459

460

461

462

463

464

465

466

467

468

469

470

471

472

473

474

475

476

477

478

479

480

Boughton, D. A. 2000. The dispersal system of a butterfly: A test of source-sink theory suggests the intermediate-scale hypothesis. American Naturalist 156:131-144.

Bullock, J. M. and R. T. Clarke. 2000. Long distance seed dispersal by wind: measuring and modelling the tail of the curve. Oecologia 124:506-521.

Chapman, D. S., C. Dytham, and G. S. Oxford. 2007. Modelling population redistribution in a leaf beetle: an evaluation of alternative dispersal functions. Journal of Animal Ecology 76:36-44.

Clobert, J., J. F. Le Galliard, J. Cote, S. Meylan, and M. Massot. 2009. Informed dispersal, heterogeneity in animal dispersal syndromes and the dynamics of spatially structured populations. Ecology Letters 12:197-209.

Comins, H. N. 1982. Evolutionarily stable strategies for localized dispersal in 2 dimensions. Journal of Theoretical Biology 94:579-606.

Comins, H. N. and M. P. Hassell. 1987. The dynamics of predation and competition in patchy environments.

Enfjall, K. and O. Leimar. 2009. The evolution of dispersal - the importance of information about population density and habitat characteristics. Oikos 118:291-299.

Ezoe, H. 1998. Optimal dispersal range and seed size in a stable environment. Journal of Theoretical Biology 190:287-293.

Fowler, H. G. 1988. Dispersal of early instars of the mole cricket, Scapteriscus tenuis, as a function of density, food, and pathogens (Orthoptera, Gryllotalpidae). Entomologia Generalis 13:15-20.

Frank, K. and C. Wissel. 1998. Spatial aspects of metapopulation survival - from model results to rules of thumb for landscape management. Landscape Ecology 13:363-379.

Frank, S. A. 1986. Dispersal Polymorphisms in Subdivided Populations. Journal of Theoretical Biology 122:303-309. 
481

482

483

484

485

486

487

488

489

490

491

492

493

494

495

496

497

498

499

500

501

502

503

504

505

506

Fuchs, E., W. Kutsch, and A. Ayali. 2003. Neural correlates to flight-related densitydependent phase characteristics in locusts. Journal of Neurobiology 57:152-162.

Gandon, S. and Y. Michalakis. 2001. Multiple causes of the evolution of dispersal. Pages 155167 in J. Clobert, E. Danchin, A. A. Dhondt, and J. D. Nichols, editors. Dispersal. oxford University Press, New York.

Getz, W. M. and D. Saltz. 2008. A framework for generating and analyzing movement paths on ecological landscapes. Proceedings of the National Academy of Sciences of the United States of America 105:19066-19071.

Gilbert, M., J. C. Gregoire, J. F. Freise, and W. Heitland. 2004. Long-distance dispersal and human population density allow the prediction of invasive patterns in the horse chestnut leafminer Cameraria ohridella. Journal of Animal Ecology 73:459-468.

Gros, A., H. J. Poethke, and T. Hovestadt. 2006. Evolution of local adaptations in dispersal strategies. Oikos 114:544-552.

Hamilton, W. D. and R. M. May. 1977. Dispersal in stable habitats. Nature 269:578-581.

Hanski, I. 1994. A practical model of metapopulation dynamics. 63:151-162.

Hassell, M. P., J. H. Lawton, and R. M. May. 1976. Patterns of dynamical behavior in singlespecies populations. Journal of Animal Ecology 45:471-486.

Hein, S., B. Pfenning, T. Hovestadt, and H. J. Poethke. 2004. Patch density, movement pattern, and realised dispersal distances in a patch-matrix landscape - a simulation study. Ecological Modelling 174:411-420.

Higgins, S. I., R. Nathan, and M. L. Cain. 2003. Are long-distance dispersal events in plants usually caused by nonstandard means of dispersal? Ecology 84:1945-1956.

Higgins, S. I. and D. M. Richardson. 1999. Predicting plant migration rates in a changing world: The role of long-distance dispersal. American Naturalist 153:464-475.

Hovestadt, T., A. Kubisch, and H. J. Poethke. 2010. Information processing in models for density-dependent emigration: A comparison. Ecological Modelling 221:405-410. 
Hovestadt, T., S. Messner, and H. J. Poethke. 2001. Evolution of reduced dispersal mortality and 'fat-tailed' dispersal kernels in autocorrelated landscapes. Proceedings of the Royal Society of London Series B-Biological Sciences 268:385-391.

Hovestadt, T. and H. J. Poethke. 2006. The control of emigration and its consequences for the survival of populations. Ecological Modelling 190:443-453.

Ichiki, R. T., Y. Kainoh, Y. Yamawaki, and S. Nakamura. 2011. The parasitoid fly Exorista japonica uses visual and olfactory cues to locate herbivore-infested plants. Entomologia Experimentalis et Applicata 138:175-183.

Janosi, I. M. and I. Scheuring. 1997. On the evolution of density dependent dispersal in a spatially structured population model. Journal of Theoretical Biology 187:397-408.

Kallimanis, A. S., W. E. Kunin, J. M. Halley, and S. P. Sgardelis. 2006. Patchy disturbance favours longer dispersal distance. Evolutionary Ecology Research 8:529-541.

Katul, G. G., A. Porporato, R. Nathan, M. Siqueira, M. B. Soons, D. Poggi, H. S. Horn, and S. A. Levin. 2005. Mechanistic analytical models for long-distance seed dispersal by wind. American Naturalist 166:368-381.

Kisdi, E. 2004. Conditional dispersal under kin competition: extension of the Hamilton-May model to brood size-dependent dispersal. Theoretical Population Biology 66:369-380.

Lambin, X., J. Aars, and S. B. Pierney. 2001. Dispersal, intraspecific competition, kin competition and kin facilitation: a review of the empirical evidence. Pages 110-122 in J. Clobert, E. Danchin, A. A. Dhondt, and J. D. Nichols, editors. Dispersal. Oxford University Press, New York.

Levin, S. A., D. Cohen, and A. Hastings. 1984. Dispersal Strategies in patchy environments. Theoretical Population Biology 26:165-191.

Monkkonen, M., R. Hardling, J. T. Forsman, and J. Tuomi. 1999. Evolution of heterospecific attraction: using other species as cues in habitat selection. Evolutionary Ecology 13:91-104. 
533 Morris, D. W. 2003. Toward an ecological synthesis: a case for habitat selection. Oecologia

534 136:1-13.

535 Morris, D. W. and J. T. MacEachern. 2010. Active density-dependent habitat selection in a

$536 \quad$ controlled population of small mammals. Ecology 91:3131-3137.

537 Motro, U. 1991. Avoiding inbreeding and sibling competition - the evolution of sexual

538 dimorphism for dispersal. American Naturalist 137:108-115.

539 Muller-Landau, H. C., S. A. Levin, and J. E. Keymer. 2003. Theoretical perspectives on

540 evolution of long-distance dispersal and the example of specialized pests. Ecology

$541 \quad \mathbf{8 4 : 1 9 5 7 - 1 9 6 7 .}$

542 Murrell, D. J., J. M. J. Travis, and C. Dytham. 2002. The evolution of dispersal distance in $543 \quad$ spatially-structured populations. Oikos 97:229-236.

544 Nathan, R. 2006. Long-distance dispersal of plants. Science 313:786-788.

545 Nathan, R., W. M. Getz, E. Revilla, M. Holyoak, R. Kadmon, D. Saltz, and P. E. Smouse.

546 2008. A movement ecology paradigm for unifying organismal movement research.

547 Proceedings of the National Academy of Sciences of the United States of America

$548 \quad$ 105:19052-19059.

549 Nathan, R., G. G. Katul, H. S. Horn, S. M. Thomas, R. Oren, R. Avissar, S. W. Pacala, and S.

550 A. Levin. 2002. Mechanisms of long-distance dispersal of seeds by wind. Nature

$551 \quad 418: 409-413$.

552 Nichols, R. A. and G. M. Hewitt. 1994. The genetic consequences of long-distance dispersal

553 during colonization. Heredity 72:312-317.

554 Pajunen, V. I. and I. Pajunen. 2003. Habitat selection in rock pool corixids: the effect of local 555 density on dispersal. Hydrobiologia 495:73-78.

556 Parvinen, K., U. Dieckmann, M. Gyllenberg, and J. A. J. Metz. 2003. Evolution of dispersal

557 in metapopulations with local density dependence and demographic stochasticity.

558 Journal of Evolutionary Biology 16:143-153. 
559

560

561

562

563

564

565

566

567

568

569

570

571

572

573

574

575

576

577

578

579

580

581

582

583

Perrin, N. and J. Goudet. 2001. Inbreeding, kinship, and the evolution of natal dispersal. Pages 123-142 in J. Clobert, E. Danchin, A. A. Dhondt, and J. D. Nichols, editors. Dispersal. Oxford University press, New York.

Pfeiffer, M., H. Huttenlocher, and M. Ayasse. 2010. Myrmecochorous plants use chemical mimicry to cheat seed-dispersing ants. Functional Ecology 24:545-555.

Poethke, H. J. and T. Hovestadt. 2002. Evolution of density-and patch-size-dependent dispersal rates. Proceedings of the Royal Society of London Series B-Biological Sciences 269:637-645.

Poethke, H. J., B. Pfenning, and T. Hovestadt. 2007. The relative contribution of individual and kin selection to the evolution of density-dependent dispersal rates. Evolutionary Ecology Research 9:41-50.

Rankin, M. A. and J. C. A. Burchsted. 1992. The cost of migration in insects. Annual Review of Entomology 37:533-559.

Rousset, F. and S. Gandon. 2002. Evolution of the distribution of dispersal distance under distance-dependent cost of dispersal. Journal of Evolutionary Biology 15:515-523.

Ruxton G.D. and P. Rohani. 1999. Fitness-dependent dispersal in metapopulations and its consequences for persistence and synchrony. Journal of Animal Ecology 68: 530-539

Roy, D. B., P. Rothery, D. Moss, E. Pollard, and T. J.A. 2001. Butterfly numbers and weather: predicting historical trends in abundance and the future effects of climate change. Journal of Animal Ecology 70:201-217.

Savill, N. J. and P. Hogeweg. 1998. Spatially induced speciation prevents extinction: the evolution of dispersal distance in oscillatory predator-prey models. Proceedings of the Royal Society of London Series B-Biological Sciences 265:25-32.

Savill, N. J. and P. Hogeweg. 1999. Competition and dispersal in predator-prey waves. Theoretical Population Biology 56:243-263. 
584 Stamps, J. A., V. V. Krishnan, and M. L. Reid. 2005. Search costs and habitat selection by

585 dispersers. Ecology 86:510-518.

586 Stamps, J. and V. V. Krishnan. 2005. Nonintuitive cue use in habitat selection. Ecology

$587 \quad \mathbf{8 6}: 2860-2867$.

588 Starrfelt, J. and H. Kokko. 2010. Parent-offspring conflict and the evolution of dispersal

589 distance. American Naturalist 175:38-49.

590 Tackenberg, O., P. Poschlod, and S. Bonn. 2003. Assessment of wind dispersal potential in

591 plant species. Ecological Monographs 73(2):191-205.

592 Travis, J. M. J. and C. Dytham. 1999. Habitat persistence, habitat availability and the

593 evolution of dispersal. Proceedings of the Royal Society of London Series B-

$594 \quad$ Biological Sciences 266:723-728.

595 Travis, J. M. J., D. J. Murrell, and C. Dytham. 1999. The evolution of density-dependent

596 dispersal. Proceedings of the Royal Society of London Series B-Biological Sciences

$597 \quad 266: 1837-1842$.

598 Travis, J.M.J., H.S. Smith, and S.M.W Ranwala. 2010. Towards a mechanistic understanding

599 of dispersal evolution in plants: conservation implications. Diversity and Distributions

600 16: $690-702$.

601 Van Dyck, H. and M. Baguette. 2005. Dispersal behaviour in fragmented landscapes: Routine

$602 \quad$ or special movements? Basic and Applied Ecology 6:535-545.

603 Zera, A. J. and R. F. Denno. 1997. Physiology and ecology of dispersal polymorphism in

604 insects. 42:207-230.

605 Zera, A. J. and L. G. Harshman. 2001. The physiology of life history trade-offs in animals.

$606 \quad$ Annual Review of Ecology and Systematics 32:95-126.

607

608 


\section{Figure Captions}

610

611 Figure 1

612 Symbolic representation of the landscape and dispersal behaviour of two representative 613 individuals. Circles represent patches of suitable habitat, the space in between the hostile 614 matrix. Clusters of circles of similar shading represent clusters of patches with similar 615 environmental conditions (for this case $4 \times 4=16$ patches per cluster). Thin arrows depict 616 possible dispersal moves during natal dispersal of a focal individual (nearest neighbour 617 dispersal). For natal dispersal an individual chooses a direction at random (fat arrow) and in 618 the following steps it keeps this direction (broken arrows).

$620 \quad$ Figure 2

621 Influence of dispersal mortality $(\mu)$ on natal emigration probability (a) and the mean number 622 of steps an emigrant takes (b), for uninformed dispersal $\left(\mathrm{DI}_{\mathrm{n}} / \mathrm{DI}_{\mathrm{f}}\right.$; filled circles), density 623 dependent natal dispersal but density independent dispersal for any subsequent dispersal steps $624\left(\mathrm{DD}_{\mathrm{n}} / \mathrm{DI}_{\mathrm{f}}\right.$; grey circles), and completely density dependent dispersal ( $\mathrm{DD}_{\mathrm{n}} / \mathrm{DD}_{\mathrm{f}}$; open circles). 625 Result of simulation experiments in worlds of 576 patches of capacity $K=40$ with 626 uncorrelated environmental fluctuations (cluster-size $k=1$ ).

628 Figure 3

629 Mean stepwise difference in population density between start- and target-patch for the first 630 five consecutive dispersal steps of dispersing individuals following different dispersal 631 strategies $\left(\mathrm{DI}_{\mathrm{n}} / \mathrm{DI}_{\mathrm{f}}=\right.$ filled circles; $\mathrm{DD}_{\mathrm{n}} / \mathrm{DI}_{\mathrm{f}}=$ grey circles; $\mathrm{DD}_{\mathrm{n}} / \mathrm{DD}_{\mathrm{f}}=$ open circles $)$. Left

632 column $(a, c)$ gives results for low dispersal mortality $(\mu=0.025)$, and right column $(b, d)$ for 633 high dispersal mortality $(\mu=0.200)$. Upper row $(a, b)$ gives results for uncorrelated (cluster- 
634 size $k=1$ ) lower column (c, d) those for correlateted (cluster-size $k=16$ ) environmental 635 conditions. Result of simulation experiments in worlds of 576 patches of capacity $K=40$. In 636 any generation, patches within same cluster are exposed to identical environmental conditions $637\left(\lambda_{t}\right)$

638

639 Figure 4

640 Influence of dispersal mortality $(\mu)$ on natal emigration probability (a) and the mean number 641 of steps an emigrant covers (b) for completely uncorrelated worlds ( $k=1$; filled circles), 642 spatial correlation distance $l=2(k=4$; grey circles $)$ and spatial correlation distance $l=4(k=$ 643 16; open circles). Result of simulation experiments with density independent dispersal $644\left(\mathrm{DI}_{\mathrm{n}} / \mathrm{DI}_{\mathrm{f}}\right)$ in worlds of 576 patches of capacity $K=40$. In any generation, patches within same 645 cluster are exposed to identical environmental conditions $\left(\lambda_{t}\right)$. 


\section{Figure 1}

647

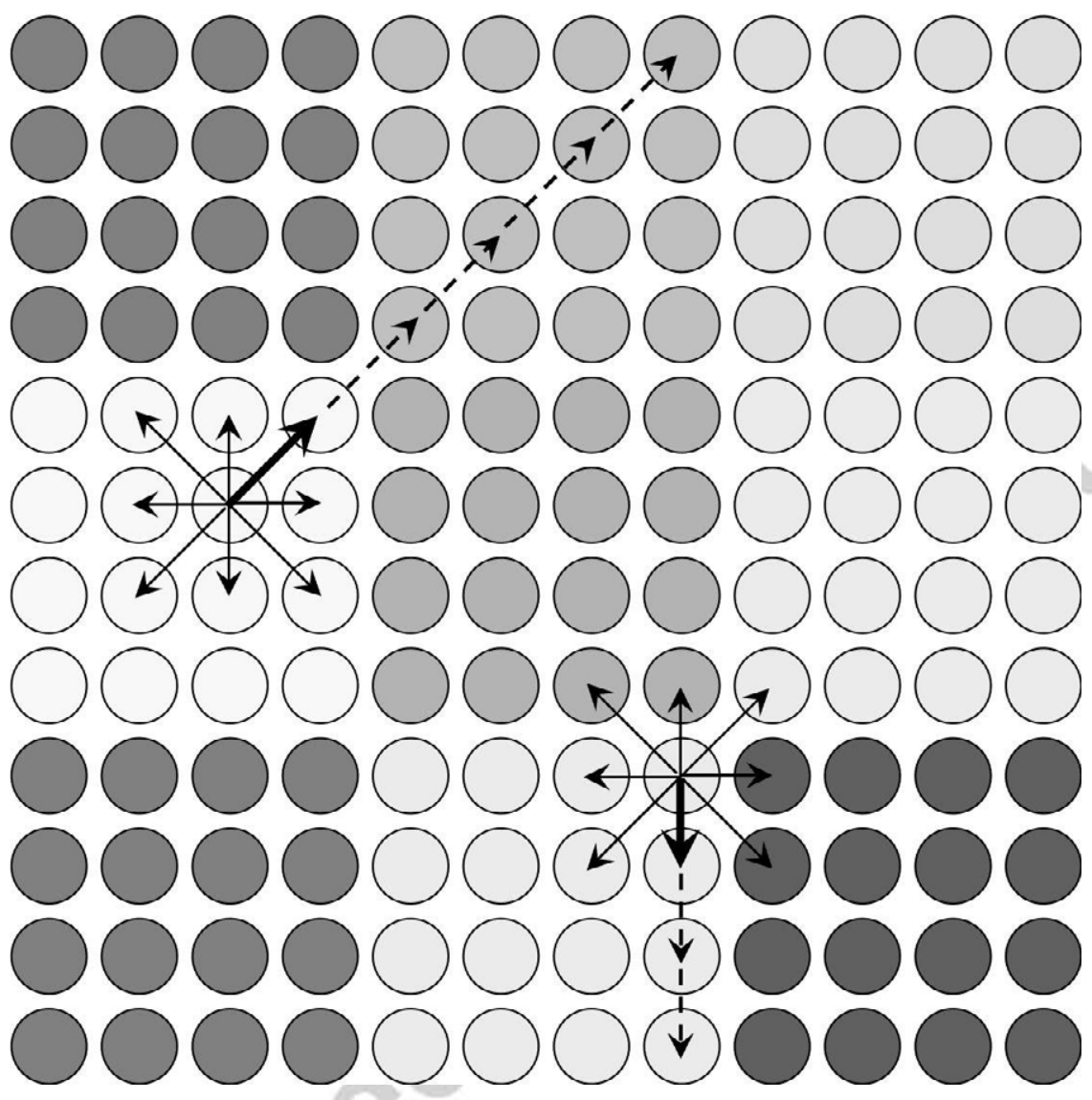

649 
$650 \quad$ Figure 2

651

652

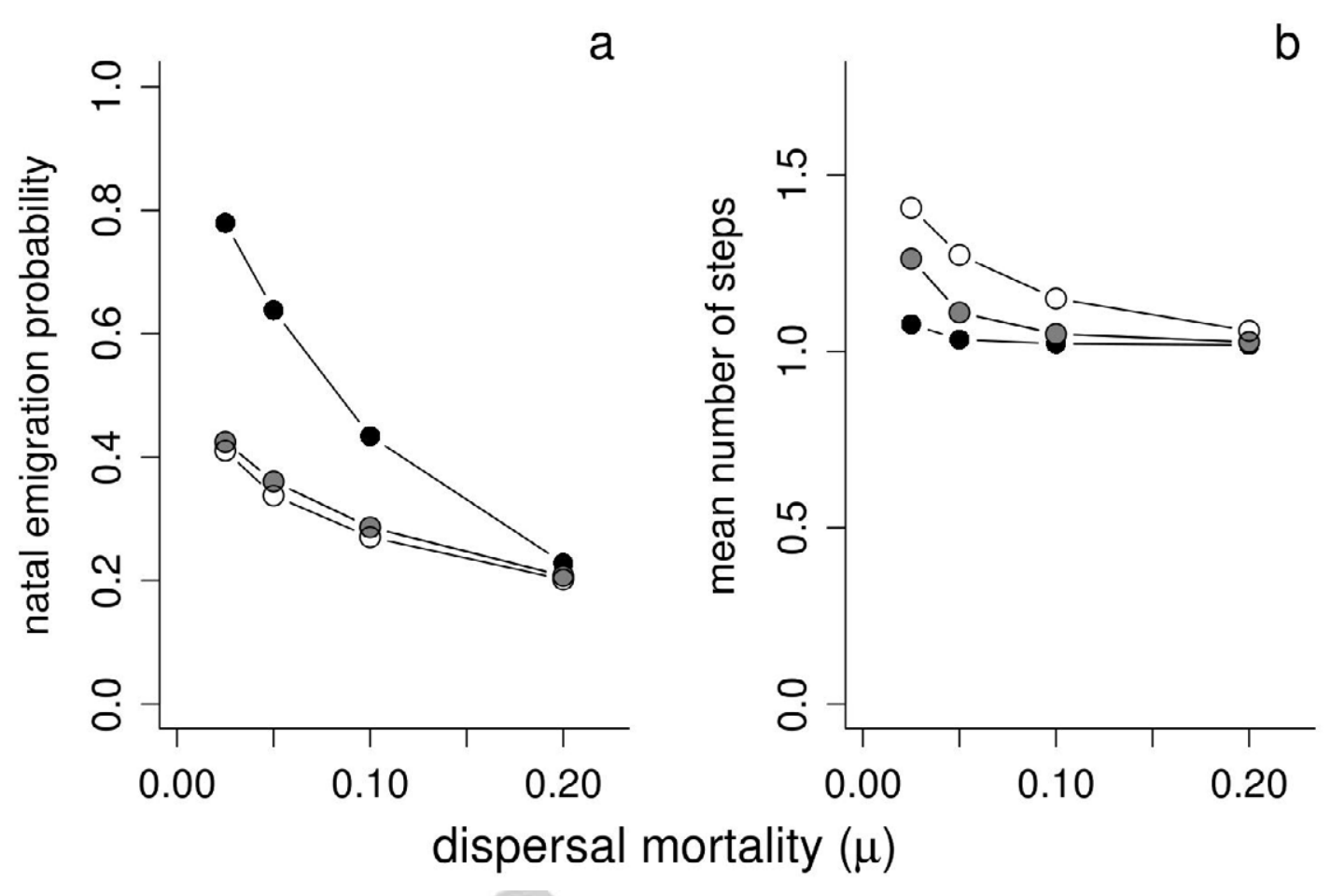

653

654 
$655 \quad$ Figure 3

656

657

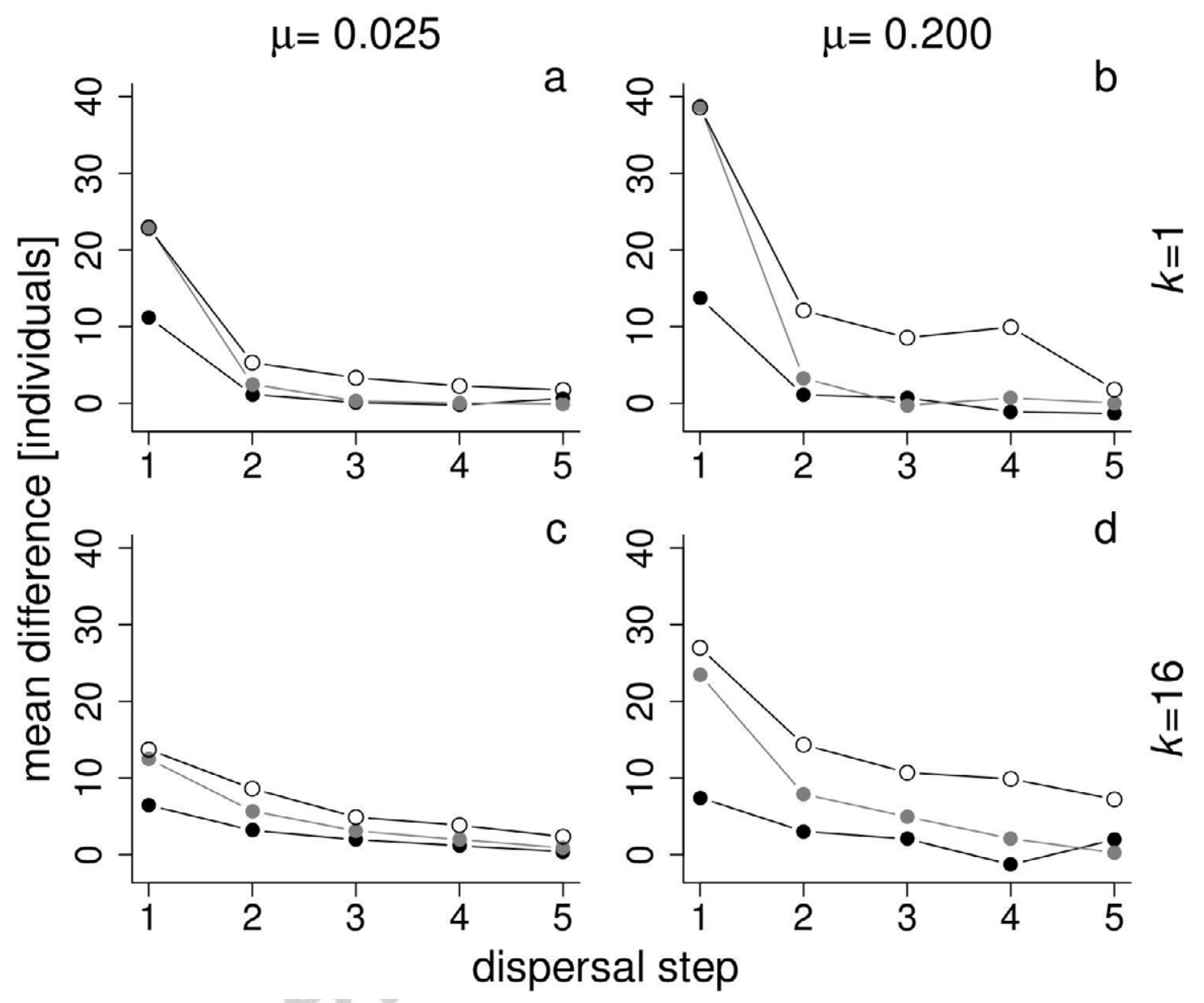

658

659

660 
$661 \quad$ Figure 4

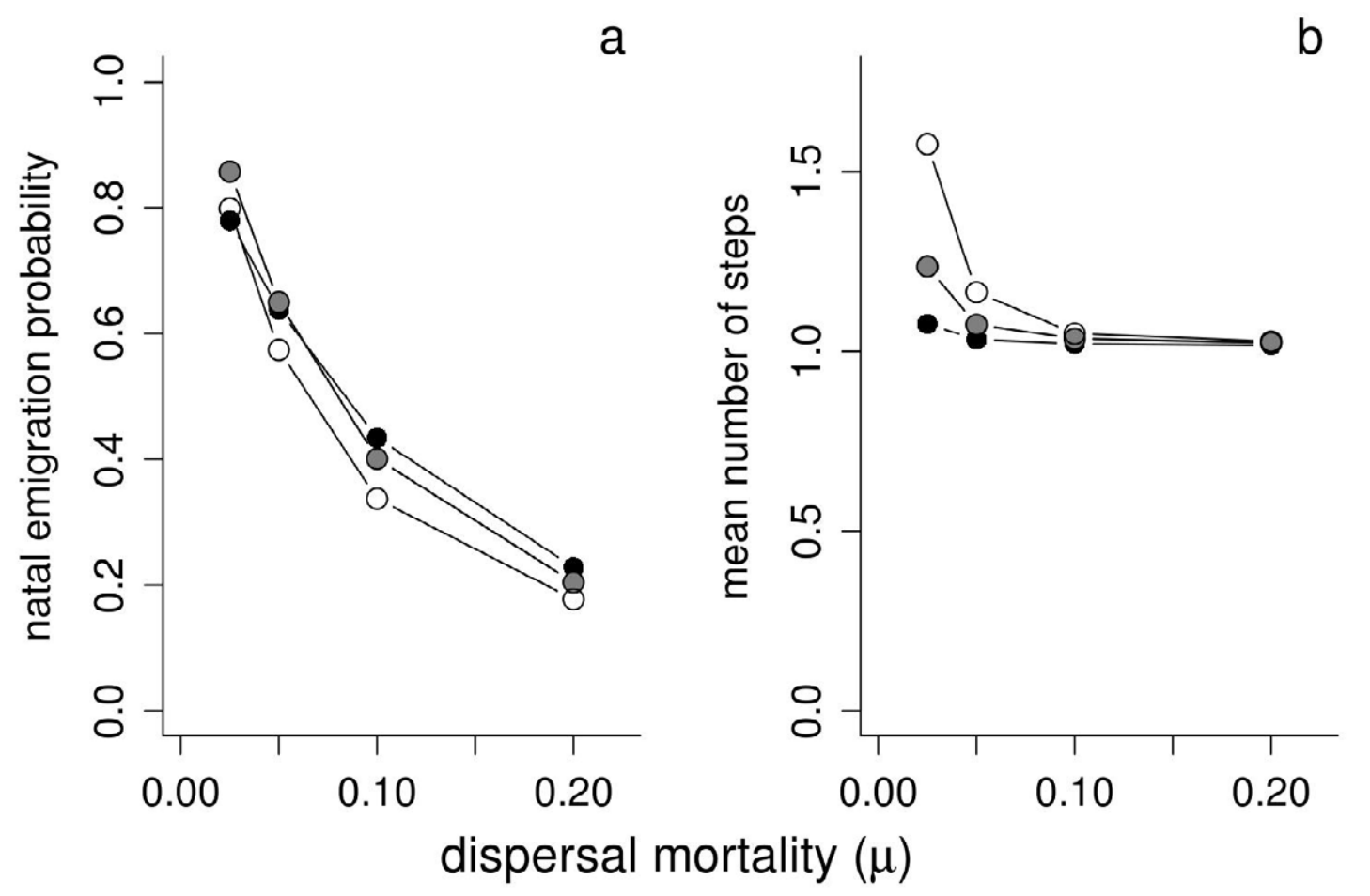

662

663

664

665 